\title{
Percevoir et reconnaître des symboles : La théorie de la représentation iconique de Dominic Lopes
}

\author{
Par MAUD HAGELSTEIN \\ FRS-FNRS, Université de Liège
}

Résumé Dans ses travaux sur la valeur épistémique des images (Understanding Pictures, 1996), Dominic Lopes (Département de philosophie, Université de Colombie Britannique) développe une esthétique analytique essentiellement tournée vers la représentation iconique, pour la compréhension de laquelle il confronte généralement deux modèles. Selon le premier de ces modèles, inspiré d'une approche perceptuelle comme celle de Richard Wollheim, les images dépendent de processus perceptifs (le perceptualisme repose sur l'idée d'une « ressemblance» entre l'image et ce qu'elle représente). Le défaut de ce modèle serait néanmoins de se fier de manière trop naïve au caractère naturel de la perception. La représentation iconique ne peut pas être expliquée par la seule perception, la vision étant bien entendu informée par les dimensions culturelle et surtout cognitive. En ce sens - et il faudra dans les lignes qui suivent pouvoir montrer pourquoi - , la reconnaissance iconique diffère de la reconnaissance visuelle ordinaire. Selon le deuxième modèle, qui mobilise les outils d'une approche symbolique comme celle de Nelson Goodman, la représentation iconique fonctionne en analogie avec d'autres types de symboles, notamment linguistiques. Une telle approche part des similitudes entre les images et le langage, en tant que représentations fonctionnant semblablement par dénotation et prédication. Or, on connait les résistances actuelles des théoriciens de l'image à l'égard du paradigme langagier : le risque d'une confrontation avec le langage serait - selon certains défenseurs de l'iconic turn - de perdre la densité sémantique de l'image, au profit d'une « simple » capacité descriptive. Entre ces deux voies, D. Lopes développe sa «théorie de la reconnaissance d'aspect», qui incorpore des éléments perceptifs à une structure de compréhension symbolique. Si les images appartiennent bien à des systèmes symboliques de dénotation, 
« il est possible qu'elles soient des symboles dont la référence dépend de l'exercice d'aptitudes perceptives ». Mon article voudrait étudier cette proposition. La théorie de Lopes permet d'expliquer l'acquisition de compétences (capacités de reconnaître) par les images, et de montrer comment les images installent progressivement ces compétences.

\section{Iconologie et élitisme (introduction)}

Si l'imagination se définit comme faculté de « voir comme» (seeing as), on s'intéressera ici à la possibilité de voir et de comprendre une image artistique $^{1}$ comme représentation d'une chose (situation, événement, individu). Partant, la question décisive sera de savoir si le « voir comme» requiert de la part du spectateur de l'œuvre une compétence cognitive (avons-nous besoin des concepts et du langage pour développer cette capacité de «voir comme » ?), ou une compétence essentiellement perceptive (sommes-nous semblablement appareillés pour percevoir les images ?), ou encore une compétence médiane. J'envisagerai donc du point de vue du récepteur l'expérience qui consiste à appréhender l'image, en particulier artistique.

Le champ problématique dans lequel s'inscrit la présente proposition est celui de l'iconologie, la méthode d'interprétation des images développée par l'historien de l'art Erwin Panofsky ${ }^{2}$. La méthode iconologique est basée sur une opération de compréhension et de lecture de l'œuvre artistique, visant à dégager progressivement les couches de signification qui la constituent, depuis le niveau formel de la signification primaire - qui recouvre les significations factuelle et expressive, jusqu'au niveau de la signification intrinsèque, aussi appelée par Panofsky le «contenu». Ainsi décrite, l'iconologie s'envisage donc comme pouvoir d'identification du contenu des œuvres. Le travail interprétatif suppose manifestement une progressivité dans l'analyse de l'œuvre : on finit d'ailleurs par écarter de l'analyse les préoccupations formelles pour se concentrer prioritairement sur les liens de l'œuvre

\footnotetext{
${ }^{1}$ Entendre l'image au sens de picture ou d'" image matérielle », acception développée entre autres par W.J.T. Mitchell. Cf. Iconology : Image, Text, Ideology, Chicago, University of Chicago Press, 1986 ; Picture Theory. Essays on Verbal and Visual Representation, Chicago, University of Chicago Press, 1994; What do Pictures Want? The Lives and Loves of Images, Chicago, University of Chicago Press, 2005.

${ }^{2}$ Cf. E. Panofsky, Essais d'iconologie: thèmes humanistes dans l'art de la Renaissance (Studies in Iconology, 1939), trad. angl. C. Herbette et B. Teyssèdre, Paris, Gallimard, 1967. E. Panofsky, L'Euvre d'art et ses significations (Meaning in the Visual Arts, 1955), trad. angl. par M. et B. Teyssèdre, Paris, Gallimard, 1969.
} 
aux symboles et aux idées. Partant, l'iconologie attire aujourd'hui les foudres de certains détracteurs (ils sont nombreux dans le champ de la théorie de l'image contemporaine), précisément en vertu de son logocentrisme apparent et de son déni supposé de l'expérience perceptive au profit d'une lecture du sens de l'œuvre finalement indifférente aux spécificités du médium ${ }^{1}$. Par ailleurs, on envisagera ici la question - trop souvent négligée - du caractère élitiste de l'iconologie dans sa version panofskienne, et plus largement de l'élitisme inhérent aux diverses iconologies (puisqu'il y a aujourd'hui de nombreuses théories vouées à comprendre la logique des images).

Les enjeux de ce problème sont non seulement épistémologiques mais encore socio-politiques, puisque, dès lors que l'on parle de facultés ou de compétences, il faut envisager la possibilité d'un partage inégal de ces compétences. Pour ouvrir ce nouveau champ problématique, qu'il ne s'agira pas d'épuiser ici, je voudrais faire une référence rapide aux réflexions de Pierre Bourdieu dans le texte introductif de La distinction $(1979)^{2}$. Ces thèses

\footnotetext{
${ }^{1}$ Voir par exemple: G. Didi-Huberman, Devant l'image, Paris, Minuit, 1990 ; G. Boehm, «Jenseits der Sprache? Anmerkungen zur Logik der Bilder», Iconic Turn. Die Neue Macht der Bilder, ed. Christa Maar and Hubert Burda, Cologne, 2004, p. 28-43 ; G. Boehm, « Die Bilderfrage», Was ist ein Bild?, ed. Gottfried Boehm, Munich 1994. Si l'on tente de rapporter la théorie de l'image allemande contemporaine (Bildwissenschaft) — telle qu'elle se déploie considérablement depuis quelques années - à l'iconologie, on doit effectivement admettre que le modèle iconologique joue surtout négativement. Depuis la fin des années 1980, la méthode iconologique a commencé à générer chez les théoriciens de l'art et du visuel (en général) toutes sortes de réactions d'insatisfaction — voire même de franche hostilité. Pour la plupart de ces auteurs, l'iconologie sert de repoussoir autant que de modèle. Les critiques généralement adressées par les théoriciens contemporains à la méthode iconologique s'articulent le plus souvent autour du problème de la prédominance du paradigme langagier pour l'analyse des images. À force de considérer trop strictement l'image sur le mode du signe qu'il faut lire et décrypter, on risque de l'assimiler purement et simplement à d'autres types de signes, notamment langagiers, et de diluer sa spécificité dans une théorie symbolique trop large. Globalement, il me semble que les différentes réactions s'accordent (ou en tout cas pourraient s'accorder) sur le fait que l'iconologie - telle qu'elle se présente dans sa version traditionnelle - doit être complétée : l'approche symbolique des œuvres doit par exemple pouvoir s'appuyer sur une attention soutenue à leurs modes de présentation (dans ce cas, les outils du «formalisme esthétique » peuvent alors être convoqués) ou à leur matérialité (les outils «phénoménologiques » peuvent idéalement venir au secours de l'analyse).

${ }^{2}$ P. Bourdieu, La distinction. Critique sociale du jugement, Paris, Minuit, coll. « Le sens commun », 1979, p. II.
} 
ne relèvent pas d'une critique de Panofsky, mais il s'agit au contraire, pour Bourdieu, d'y voir une confirmation (anticipée) de sa propre proposition, selon laquelle l'art, ou plus largement le domaine des artefacts culturels, génère, plus encore que d'autres champs symboliques, des phénomènes de distinction, des phénomènes discriminants, «classants »- le domaine des préférences artistiques étant pour l'individu un lieu d'affirmation de sa valeur et de sa position au sein de l'espace social. On s'en rappelle, Bourdieu a contribué à introduire les idées de Panofsky en France; il a notamment accueilli dans la collection "Le sens commun » qu'il dirigeait chez Minuit, mais aussi traduit lui-même et postfacé l'ouvrage Architecture gothique et pensée scolastique ${ }^{1}$. Pour Bourdieu, la méthode iconologique démontre en effet l'utilité des codes et l'importance de l'acquisition de ces codes pour le décryptage des images artistiques. Le spectateur privé des connaissances nécessaires, c'est-à-dire privé des codes en vigueur dans le champ de la représentation picturale, se trouvera évidemment bien dépourvu face aux chefs-d'œuvre de l'art légitime : sans "code spécifique», le spectateur se sentira submergé par sa perception, et par ce qui lui apparaîtra comme un chaos sensible (rythmes, couleurs et lignes) sans raison. Dépourvu des connaissances qui lui permettraient de « donner raison » à ce magma sensible, le spectateur est forcé de s'en tenir aux propriétés sensibles ou aux résonnances affectives liées à ces propriétés (ce que Panofsky désignait comme la couche primaire du sens de l'œuvre). Il lui est par ailleurs impossible de pénétrer la couche des sens secondaires, la " région du sens du signifié », sans posséder les concepts qui lui permettraient de dépasser les propriétés sensibles pour saisir "les caractéristiques proprement stylistiques de l'œuvre». Aux yeux de Bourdieu, l'œuvre d'art authentique implique une "opération de déchiffrement» (qui est un « acte de connaissance » plutôt qu'une expérience sensible), déchiffrement qui suppose «la mise en œuvre d'un patrimoine cognitif, d'une compétence culturelle $»^{2}$. Il y a d'ailleurs une symétrie entre la hiérarchie des classes sociales et celle des couches de signification progressivement dégagées dans la saisie de l'œuvre: la perception sensible serait laissée aux classes inférieures, quand la compréhension du sens authentique de l'œuvre serait réservée - de par leurs savoirs accumulés — aux classes les plus cultivées ${ }^{3}$.

${ }^{1}$ E. Panofsky, Architecture gothique et pensée scolastique (1951), Paris, Minuit, coll. « Le sens commun », 1967.

${ }^{2}$ P. Bourdieu, La distinction. Critique sociale du jugement, op. cit., p. 16-29.

${ }^{3}$ D'où la question que l'on pourrait adresser à la théorie de Husserl (cf. article de A. Dufourcq dans ce volume): si l'on pense qu'il est impossible de repousser 
Le caractère apparemment logo-centré de l'iconologie ${ }^{1}$ dans sa fondation panofskienne explique aussi pourquoi l'actualisation de l'iconologie passe depuis les années 1990 par une série de débats articulés autour de l'iconic turn (le tournant iconique), à savoir l'idée qu'un changement de paradigme aurait fait basculer la prédominance du langagier pour l'analyse des images au profit d'une approche désormais centrée sur les spécificités aussi bien logiques que sensibles - du visuel. Ce débat a été très vif en Allemagne durant ces années et a contribué à fonder la Bildwissenschaft ${ }^{2}$. En effet, généralement opposés à la sémiotisation excessive des formes d'expression visuelles, ces théoriciens s'accordent pour affirmer la possibilité d'une fonction intransitive de l'image, autorisant à voir l'image comme image, et réclamer un retour à l'expérience perceptive dans son épaisseur propre. Ces débats envisagent de très près les éléments qui permettent de distinguer entre les formes d'expression de l'image et du langage.

l'émergence de l'objet-image à partir de la chose-image (il serait donc impossible de ne voir que des lignes ou des couleurs devant une toile), comment expliquer ces situations problématiques où l'on n'est pas en mesure de décrypter l'œuvre (en l'absence de codes) et où le contenu nous échappe ? Et que faire des identifications fausses, des horizons d'objectivation de la chose qui seraient mal définis ?

${ }^{1}$ Un autre chantier m'amène néanmoins à relativiser cette critique. Voir notamment : A. Rieber, Art, histoire et signification. Un essai d'épistémologie d'histoire de l'art autour de l'iconologie d'Erwin Panofsky, Paris, L'Harmattan, 2012.

${ }^{2}$ Sur la Bildwissenschaft, voir la sélection de textes en cours de traduction pour une anthologie à paraître aux éditions Mimesis (Bildwissenschaft. La "science de l'image " dans les débats contemporains, textes traduits et commentés par M. Hagelstein et C. Letawe, à paraître en 2017) : H. Belting, « Echte Bilder und falsche Körper - Irrtümer über die Zukunft des Menschen », in Christa Maar/Hubert Burda (ed.), Iconic turn. Die neue Macht der Bilder, DuMont, 2004 ; K. Sachs-Hombach, « Das Bild in der Spannung von perzeptuellen und semiotischen Determinanten », in K. Sachs-Hombach, Bildwissenschaft. Zwischen Reflexion und Anwendung, Halem, 2005; L. Wiesing, « Methoden der Bildwissenschaft», in K. Sachs-Hombach, Bildwissenschaft. Zwischen Reflexion und Anwendung, Halem, 2005 ; B. Waldenfels, "Ordnungen des Sichtbaren », in G. Boehm, Was ist ein Bild?, Fink, 1994 ; K. Sachs-Hombach, J.R.J. Schirra, « Medientheorie, visuelle Kultur und Bildanthropologie », in K. Sachs-Hombach, Bildtheorien. Anthropologische und kulturelle Grundlagen des visualistic turn, Suhrkamp, 2009 ; L. Wiesing, " Sind Bilder Zeichen? », in K. Sachs-Hombach, K. Rehkämper, Bild Bildwahrnehmung - Bildverarbeitung, DUV, 1998; H. Bredekamp, «Drehmomente - Merkmale und Ansprüche des iconic turn», in C. Maar, H. Burda (ed.), Iconic turn. Die neue Macht der Bilder, DuMont, 2004. 
On quittera ici l'environnement intellectuel européen pour étudier une théorie qui redistribue autrement les problèmes nés dans le sillage de l'iconologie. Dans ses travaux sur la valeur épistémique des images (en particulier Understanding Pictures) ${ }^{1}$, Dominic McIver Lopes développe une esthétique analytique essentiellement tournée vers la représentation iconique ou « dépiction » (depiction) - terme qui recouvre la production et la compréhension d'images matérielles, démotiques ou artistiques. Après avoir reconnu les limites des modèles (1) perceptuel et (2) symbolique pour la compréhension de l'image, Lopes construit, sur base du concept de (3) " sélectivité» (selectivity), qualifiant à ses yeux la spécificité du visuel, une théorie de la (4) « reconnaissance d'aspects » (aspect recognition) dont les développements indiquent de quelle façon (5) les images nous instruisent.

\section{Limites du modèle perceptuel}

À en croire la préface inédite rédigée par Lopes pour l'édition française, les thèses développées dans Understanding Pictures sont entièrement redevables à un paysage intellectuel tendu entre (et ouvert par) les figures de Richard Wollheim et de Nelson Goodman, lesquels défendent deux modèles opposés pour la compréhension de la représentation iconique.

Selon le premier de ces modèles, celui de l'approche perceptuelle de Richard Wollheim, les images dépendent de processus perceptifs pour la raison essentielle qu'une ressemblance est nécessaire entre l'image et ce qu'elle représente ${ }^{2}$.

Or, le premier défaut de la théorie selon laquelle toute image s'inscrit dans une économie de la ressemblance serait qu'elle échoue à "rendre compte de la diversité des représentations et de la double-perception qui

\footnotetext{
${ }^{1}$ D. Lopes, Understanding Pictures, Oxford Scholarship, 1996. Traduction française de L. Blanc-Benon: Comprendre les images: une théorie de la représentation iconique, Rennes, PUR, coll. "Aesthetica», 2014. Lire aussi de Lopes : Sight and Sensibility, Oxford University Press, 2005 ; «Picture this : Image-based Demonstratives », in Catharine Abell, Katerina Bantinaki (ed.), Philosophical Perspectives on Depiction, Oxford University Press, 2010. Sur Understanding Pictures, cf. : John V. Kulvicki, On Images. Their Structure and Content, Oxford University Press, 2009. On trouvera aussi de nombreuses références aux travaux de Lopes dans : Bas C. Van Fraasen, Scientific Representation, Oxford University Press, 2008.

${ }^{2}$ Cf. par ex.: R. Wolheim, « Voir-comme, Voir-dans et la représentation picturale », in L'art et ses objets, trad. fr. R. Crevier, Paris, Aubier, 1994 ; Painting as an Art, Princeton, Princeton University Press, 1987.
} 
caractérisent la dépiction ${ }^{1}$. La double-perception (twofoldness) définit la capacité de capter d'un même regard aussi bien le sujet de l'image que son dessin (par dessin/design il faut entendre très largement la manière dont elle est faite et construite). Il existe une multitude de manières, pour une pomme peinte par exemple, de ressembler à une pomme réelle. On dit trop peu de choses si on se contente de relever - en se fiant aux indications de notre perception - une ressemblance entre l'image matérielle et son référent.

L'autre défaut de ce modèle serait de se fier de manière trop naïve au caractère naturel de la perception. La représentation iconique (la dépiction ou mise en image) ne s'explique pas par la seule perception, la vision y étant informée par les dimensions culturelle et surtout cognitive. Lopes s'attache à défaire le mythe de l'œil innocent. Pour lui, la reconnaissance iconique diffère de la reconnaissance visuelle ordinaire. Mais pour autant, la juste compréhension de l'image n'est pas prioritairement conditionnée par des savoirs antérieurs, des savoirs qui auraient été accumulés en amont à propos du sujet représenté. Pour pouvoir établir une bonne interprétation, il importe avant tout de se situer dans le bon système de représentation. Il faut pouvoir, dira Lopes, reconnaître l'aspect - expression qui articule dans sa formulation même les dimensions cognitive et perceptive : "Pour pouvoir remarquer des similarités entre une image et son sujet il faut connaitre le système de l'image, pas ce que l'image représente $»^{2}$. Cette condition particulière propre à la dépiction sert d'indicateur à ce qui la différencie qualitativement de l'expérience perceptive. Selon Lopes, il ne s'agit ni de penser l'expérience iconique comme «un cas spécial de la perception visuelle ordinaire (ordinary visual experience) » (et de considérer la continuité entre ces deux expériences), ni de les opposer radicalement (en disant que voir des choses dans des images n'aurait rien à voir avec voir des choses en chair et en os) ${ }^{3}$. La perception est bien mobilisée par l'expérience iconique, même si l'image met son spectateur dans cette situation particulière - et inédite - de devoir identifier et comprendre le système de l'image avant même d'être en mesure de saisir les ressemblances, ou plus exactement de devoir comprendre le système de l'image au moment et à travers l'identification de ressemblances.

Wollheim a raison de souligner que le fait d'être conscient simultanément du contenu représentationnel d'une image (picture's content) et de sa facture (design) est au cœur de l'expérience de nombreuses images. L'utilisation de

\footnotetext{
${ }^{1}$ D. Lopes, Understanding Pictures, op. cit., p. 55 ; trad. fr. p. 77.

${ }^{2}$ Ibid., p. 33 ; trad. fr. p. 56.

${ }^{3}$ Ibid., p. 37 ; trad. fr. p. 59.
} 
la couleur dans un tableau comme Le supplice de Marsyas du Titien instaure une tension entre la couleur appliquée sur la surface et la couleur représentée : remarquer cette tension et tenter de concilier les deux expériences fait partie intégrante de l'expérience que l'on fait d'une telle image ${ }^{1}$.

Pour le dire avec d'autres formules, Lopes soumet les théories perceptives de la dépiction (ou de la représentation iconique) à une double critique. D'une part, il en manifeste les limites, en faisant apparaître le caractère non-naturel de nos capacités à saisir des ressemblances, et il faudra préciser par quoi ces capacités sont déterminées. D'autre part, il propose d'y substituer une description inédite du rôle de la perception dans la dépiction - et j'y reviendrai au moment d'aborder la reconnaissance d'aspect.

Puisque les théories perceptives de la dépiction sont - en l'état dans une impasse, Lopes suggère de prendre au sérieux la possibilité de considérer les similitudes entre images et langage, avec toujours dans l'esprit le projet de ne pas négliger pour autant le rôle de la perception.

\section{Limites du modèle symbolique}

Selon le deuxième modèle, qui mobilise les outils d'une approche symbolique comme celle de Nelson Goodman, la représentation iconique fonctionne par analogie avec d'autres types de symboles, notamment linguistiques.

L'alternative au perceptualisme correspond à toute théorie qui souligne des analogies entre la représentation iconique et d'autres types de symboles, en particulier linguistiques. Les analogies linguistiques nous donnent accès aux outils sophistiqués de la philosophie du langage et nous permettent d'étudier ce que j'appelle la «logique» des images. Leur inconvénient est qu'elles menacent les images d'une assimilation aux descriptions ${ }^{2}$.

Car, en effet, l'insistance sur le caractère seulement descriptif de l'image laisse supposer le caractère relatif du véhicule du message, du médium (or il est évident qu'une pomme peut être peinte selon des codes picturaux très différents les uns des autres - sinon quel sens y aurait-il à reproduire inlassablement les mêmes motifs ?). Ceci dit, comme on l'a déjà évoqué au départ des thèses principales de Lopes, affirmer que les images sont des

${ }^{1}$ Ibid., p. 42 ; trad. fr. p. 64.

${ }^{2}$ Ibid., p. 11 ; trad. fr. p. 32. 
symboles (au même titre que les textes, les cartes, les diagrammes, les partitions, etc.) ne devrait pas être incompatible avec une prise en compte de la perception. Certains théoriciens accorderont volontiers aux images d'être des symboles, mais des symboles médiatisés par des processus perceptifs ${ }^{1}$.

Dans son ouvrage Languages of Art (1968) ${ }^{2}$, Nelson Goodman propose une théorie symbolique du « faire image ». Goodman insiste expressément sur l'analogie entre dépiction et langage, même si des différences de degrés sont observées : la dépiction est sémantiquement et syntaxiquement dense (plutôt que notationnelle). Il existe des systèmes qui sont seulement notationnels, comme la musique par exemple, qui fonctionne sur une correspondance terme à terme (chaque note reportée sur le papier renvoie à un son, chaque "caractère " musical possède un et un seul correspondant). Au contraire, les images seraient saturées - et donc potentiellement sources d'ambiguïté et de recouvrements. Néanmoins, selon cette théorie symbolique, les images représentent des objets, exactement à la manière dont d'autres systèmes symboliques le font, par la dénotation (qui définit l'extension d'un domaine) et la prédication au sein d'un système ${ }^{3}$. Et en réalité, la

${ }^{1}$ Ce problème n'est pas nouveau et d'autres cultures théoriques ont pu l'investir. Dans le champ de la phénoménologie française, on relira par exemple certains passages de La prose du monde, dans lesquels Merleau-Ponty envisage les liens et la spécificité relative du verbal et du visuel. Cf. «Le langage indirect» : «Puisque la même opération expressive fonctionne ici et là, il est possible de considérer la peinture sur le fond du langage et le langage sur le fond de la peinture, et c'est nécessaire si l'on veut les soustraire à notre accoutumance, à la fausse évidence de ce qui va de soi » (M. Merleau-Ponty, La prose du monde, texte établi et présenté par C. Lefort, Paris, Gallimard, 1969, p. 64). Plus loin : « Mais enfin, demandera-t-on peut-être, si vraiment la peinture était un langage, il y aurait moyen de donner dans le langage articulé un équivalent de ce qu'elle exprime à sa manière. Que dit-elle donc ? » (p. 80). « On ne peut pas plus faire l'inventaire d'une peinture - dire ce qui y est et ce qui n'y est pas - que d'un vocabulaire, et pour la même raison : elle n'est pas une somme de signes, elle est un nouvel organe de la culture humaine qui rend possible, non pas un nombre fini de mouvements, mais un type général de conduite, et qui ouvre un horizon d'investigations » (p. 84).

${ }^{2}$ N. Goodman, Languages of Art. An Approach to a Theory of Symbols, Indianapolis, Bobbs-Merrill, 1968 (2d ed. Indianapolis, Hackett, 1976). L'ouvrage est issu de conférences prononcées en 1962 sur Locke et marque le début d'un « tournant symbolique » dans la pensée de Goodman. Les thèses qui y sont développées manifestent une conception cognitive des arts, dont la fonction principale serait de nous donner à comprendre le monde et de développer des savoirs à son propos.

${ }^{3}$ Le cas des images cinématographiques transforme encore le rapport représentatif - et supposé symbolique - de l'image aux objets. Nombreux sont les théoriciens 
concession faite par Goodman à la spécificité de l'image, lorsqu'il considère que cette dernière est dense plutôt que notationnelle, concerne la référence plutôt que l'aspect. Partant, le principal reproche adressé par Lopes à la théorie symbolique de Goodman, qui fait écho aux critiques adressées par les théoriciens de l'image actuels à l'iconologie panofskienne, tient au fait que la référence d'une image (pictorial reference) ne semble dépendre en aucune manière de son mode de présentation ${ }^{1}$. Le risque serait alors de perdre la densité et la complexité sémantique de l'image (ce que Lopes appelle son « contenu »), au profit de sa seule capacité descriptive :

À présent, il devrait être clair que le fait d'affirmer que les images appartiennent à des systèmes symboliques de dénotation (denotative symbol systems) ne diminue aucunement l'intérêt des explications perceptives de la dépiction. L'intuition de Goodman selon laquelle on peut penser les images avec succès sur le modèle d'autres sortes de représentations est handicapée par une idée austère et quelque peu idiosyncratique que l'on se fait de la référence linguistique. Mais il n'y a aucune raison de se sentir lié par la conception goodmanienne de la référence. Les liens entre la référence et la perception sont plus riches que l'on aurait pu imaginer au premier abord. Si les images sont des symboles, il est possible qu'elles soient des symboles dont la référence dépend de l'exercice d'aptitudes perceptives (perceptual skills) ${ }^{2}$.

qui refusent le modèle symbolique. On peut relire en ce sens certains passages de Pasolini (qui anticipent d'ailleurs la réflexion sur la sélectivité) : "Rien n'oblige autant à regarder les choses que de faire un film. Le regard d'un écrivain sur un paysage champêtre ou urbain peut exclure une infinité de choses, en découpant de leur ensemble uniquement celles qui émeuvent ou qui sont utiles. Le regard d'un metteur en scène sur le même paysage ne peut pas, à l'inverse, ne pas prendre conscience, en dressant quasiment une liste, de toutes les choses qui s'y trouvent. En effet, alors que chez un écrivain les choses sont destinées à devenir des mots, c'est-àdire des symboles, au contraire, dans la manière de s'exprimer qui est celle d'un metteur en scène, les choses restent des choses : les "signes" du système verbal sont donc symboliques et conventionnels, tandis que les "signes" du système cinématographique sont justement les choses elles-mêmes, dans leur matérialité et leur réalité » (P. P. Pasolini, Lettres luthériennes. Petit traité pédagogique (1976), trad. fr. A. R. Pullberg, Paris, Seuil, 2000, p. 47).

${ }^{1}$ D. Lopes, Understanding Pictures, op. cit., p. 93 ; trad. fr. p. 119.

${ }^{2}$ Ibid., p. 107 ; trad. fr. p. 134. 


\title{
3. Le concept de sélectivité comme spécificité du visuel
}

Reste à savoir - Lopes partage ce problème avec les théoriciens de l'iconic turn - ce qui fonde la spécificité de l'image à l'égard du langage, étant donné le caractère relativement insatisfaisant du critère de saturation. Depuis trois décennies au moins, on a décrit cette différence structurelle et logique de l'image et du langage de plusieurs manières, qui peuvent toutes se rapporter à l'idée communément partagée selon laquelle « une image vaut mieux qu'un long discours ».

\begin{abstract}
Il est naturel d'avoir des réticences à utiliser le langage comme modèle pour penser la dépiction. Les comparaisons possibles entre les images et le langage entrent en contradiction flagrante avec nos intuitions concernant ce qui les différencie. Mais personne ne prétend que les images sont exactement du même ordre que les énoncés linguistiques (linguistic utterances). (...) Que les images soient comparables au langage sous certains aspects déterminants ne doit pas nécessairement aller contre l'impression que nous pouvons avoir par ailleurs de ce qui les différencie ${ }^{1}$.
\end{abstract}

Les images peuvent effectivement être rapportées ou comparées au langage en tant qu'elles sont des « véhicules de stockage, manipulation et communication d'information ». Elles partagent avec le langage la charge de «représenter le monde et les pensées que nous avons à son sujet »- ceci étant plus valable encore en ce qui concerne les images démotiques ${ }^{2}$.

Alors même qu'ils s'inscrivent semblablement dans le champ symbolique, le langage et la dépiction développeraient des modalités différentes en écart l'une avec l'autre. La théorie de Goodman permet par exemple de tester l'idée selon laquelle la dépiction serait analogique tandis que le langage serait digital. À suivre cette thèse, pour ce qui concerne la dépiction, chaque transformation de l'image (chaque intervention dans sa matérialité, dans sa facture) induirait une variation analogue de son sens. Chaque transformation perçue par le regard serait convertie de manière directe, et par conséquent - les significations symboliques seraient strictement représentatives de leur source sensible. Elles se conçoivent symétriquement. Pour le langage, à l'inverse, le code détermine l'usage et protège le sens des micro-variations parasitaires; l'argument classique et pourtant discutable étant que la variation de la taille de police n'affecte pas le message transmis.

${ }^{1}$ Ibid., p. 56 ; trad. fr. p. 78

${ }^{2}$ Ibid., p. 7 ; trad. fr. p. 28. 
Loin d'être convaincu par cette proposition, Lopes travaille à déconstruire le mythe de la spécificité analogique de l'iconique, montrant par exemple que tous les systèmes iconiques ne s'inscrivent pas forcément dans le régime analogique :

$\mathrm{Au}$ contraire, comme l'a souligné Kent Bach, rien n'exclut un système d'images composé d'un répertoire restreint de points de tailles et de couleurs différentes - imaginons par exemple un système constitué d'images composées de points Ben Day en couleur à la Lichtenstein. Un tel système ne serait pas analogique si les règles pour le déchiffrer exigeaient que nous négligions les petites variations de taille, de forme ou de couleur des points, à la manière dont on demande aux lecteurs de ce livre de négliger les variations d'interligne ou l'absence d'un empattement sur une lettre parce qu'ils ne seraient pas pertinents pour déterminer quels caractères sont inscrits. De manière assez surprenante, Goodman admet qu'un système d'images notationnel, nonanalogique, pourrait surgir par le biais de la «coutume » (custom) ou d'une « stipulation expresse ». La spécificité analogique n'est donc pas essentielle à la dépiction ${ }^{1}$.

Puisque l'analogicité ne suffit pas à saisir la spécificité de la dépiction, Goodman ajoute le critère de saturation (repleteness), montrant que les images appartiennent à des systèmes plutôt (c'est-à-dire «relativement ») saturés : prenons deux systèmes, le plus saturé des deux sera celui qui aura le nombre le plus élevé de propriétés formelles (propriétés du dessin/design) pertinentes pour la représentation ${ }^{2}$. Par exemple, un électroencéphalogramme est moins saturé qu'une toile peinte parce que sa lecture n'est pas directement affectée par l'épaisseur de ses lignes. On pourrait donc croire à suivre Goodman - selon la restitution de ses idées par Lopes - que la saturation constitue un critère de spécificité pour les systèmes iconiques. Ceci dit, peut-on sans difficulté comparer les différents systèmes iconiques ? Sur quelle base ? Un système iconique serait plus saturé s'il possède davantage de propriétés pertinentes, c'est-à-dire de propriétés qui affectent — de façon analogique — le sens de la représentation. Sauf qu'une "propriété pertinente" peut avoir des effets différents dans des systèmes iconiques différents: une même propriété peut correspondre à différents contenus dans différents systèmes. Par exemple, la grandeur des figures est une indication spatiale pertinente dans une représentation «albertienne» alors qu'elle est une indication de valeur ou d'importance

${ }^{1}$ Ibid., p. 112 ; trad. fr. p. 138-139.

${ }^{2}$ Ibid., p. 113 ; trad. fr. p. 139. 
relative dans le cas de la représentation byzantine (on voit encore trace de ce modèle byzantin dans certains tableaux de Piero de la Francesca, où les personnages au premier plan sont plus petits que la Vierge). Autrement dit, des systèmes différents peuvent partager les mêmes propriétés.

Et plus encore, si la saturation est une question de degré, comment en faire une marque distinctive du caractère iconique, sans que certaines images soient considérées comme étant plus iconiques que d'autres? Ce serait évidemment absurde ; il faudrait considérer par exemple que les peintures sont plus iconiques que les dessins sous prétexte qu'elles possèdent plus de propriétés pertinentes pour la représentation.

Lopes s'attache ensuite à progressivement donner corps à sa propre théorie distinctive de la dépiction à l'égard des formes linguistiques. Pour la construire, il s'adosse notamment aux développements de F. Dretske dans Knowledge and the Flow of Information $(1981)^{1}$. Selon Dretske, l'image contient des informations analogiques par nécessité. $\mathrm{Si}$ on prend l'information "un homme est en train de faire une bulle de savon », on s'aperçoit que, transposée dans un tableau de Chardin (c'est-à-dire véhiculée par un système iconique), elle ne peut prendre qu'une forme analogique. Quand Chardin peint ce tableau, il ne peut pas faire autrement que de préciser la taille de la bulle, la manière dont elle est fabriquée (avec quel instrument ?), mais aussi le physique et l'allure vestimentaire du souffleur. L'information " un homme fait une bulle de savon » est donc augmentée et chargée de tout ce que nous pouvons apprendre en regardant la toile de Chardin, et s'il fallait traduire en mots l'information contenue dans l'image, il faudrait en donner une description extrêmement détaillée. Raison pour laquelle Dretske soutient que «l'expérience perceptive, comme les images, est analogique, ou hautement spécifique, contrairement à la cognition, qui est digitale $»^{2}$. À quoi Lopes répond que certains théoriciens (Peacocke) ont montré que le langage peut aussi être analogique - et que par ailleurs, dans le cas de certaines images très simples, une description exhaustive du contenu reste possible (il ne s'agit donc pas d'une spécificité absolue) ${ }^{3}$. Néanmoins, ces analyses rendent saillante la question de savoir quels éléments du réel sont intégrés à la représentation, quels éléments du réel sont sélectionnés.

${ }^{1}$ F. I. Dretske, Knowledge and the Flow of Information, Cambridge, The MIT Press/Bradford Books, 1981.

${ }^{2}$ D. Lopes, Understanding Pictures, op. cit., p. 115 ; trad. fr. p. 141.

${ }^{3}$ C. Peacocke, « Perceptual content », in J. Almog, J. Perry, H. K. Wettstein, Themes from Kaplan, New York, Oxford University Press, 1983. 
Selon la proposition de Lopes, la notion de sélectivité (selectivity) pourrait faire office de marque distinctive du contenu iconique à l'égard du contenu langagier. Or, il y aurait deux manières de comprendre la façon dont les images peuvent être sélectives quant à leur contenu - et seule la deuxième semble en mesure de révéler la spécificité de l'iconique. (1) On peut d'abord tenter de défendre le caractère incomplet, indéterminé et toujours partiel de la dépiction. Tel est le sens de la raillerie de Lewis Carroll : si on voulait dessiner une carte vraiment précise et parfaitement déterminée de la Grande-Bretagne, il faudrait qu'elle soit d'une taille égale à celle de la Grande-Bretagne elle-même. Cet argument est pertinent mais il s'agit aux yeux de Lopes d'une "forme de sélectivité que les images partagent avec d'autres représentations ${ }^{1}$. De la même manière, une description littéraire ne doit pas mentionner toutes les taches de rousseur d'un visage ou tous les grains de beauté d'un $\operatorname{dos}^{2}$. On peut toujours omettre - volontairement ou non - d'entrer dans certains détails. (2) Mais il y a une autre manière de penser la sélectivité dans le cas des images (qui tient aux «caractéristiques structurelles de la dépiction »). La sélectivité est alors conçue non pas comme simple omission, mais comme véritable refus. En ce sens-là :

Les images ne sont pas sélectives parce qu'elles négligent de s'engager sur certaines propriétés. Les images sont sélectives parce que, pour représenter certaines caractéristiques spatiales de leurs sujets, elles sont empêchées d'en représenter d'autres. La raison en est simplement que toutes les relations dans l'espace entre des objets à trois dimensions ne peuvent pas être représentées sur une surface à deux dimensions. Le fait d'opérer une sélection pour représenter certaines relations dans l'espace rend d'autres relations impossibles à représenter. Si cette hypothèse est correcte et que les images sont des représentations qui incarnent des aspects de leurs sujets qui incluent toujours des refus explicites d'engagement (explicit non-commitments), alors nous devrions nous attendre à pouvoir différencier les images entre elles en fonction des aspects qu'elles représentent ${ }^{3}$.

\footnotetext{
${ }^{1}$ D. Lopes, Understanding Pictures, op. cit., p. 117 ; trad. fr. p. 144.

${ }^{2}$ Voir le texte de B. Leclercq repris dans ce volume: «Pour les meinongiens, en effet, c'est une spécificité des objets réels que d'être complets, c'est-à-dire déterminés pour toute paire de prédicats (pertinents) - en objet réel, j'ai ou non un nævus sur l'épaule droite - tandis que les objets inexistants sont généralement incomplets - faute que Conan Doyle se soit prononcé sur cette question, Sherlock Holmes est indéterminé quant au fait qu'il a ou non un nævus sur l'épaule droite ».

${ }^{3}$ D. Lopes, Understanding Pictures, op. cit., p. 125 ; trad. fr. p. 152.
} 
Selon l'exemple choisi par Lopes, quand le douanier Rousseau peint un tigre, il refuse de s'engager sur la question du nombre de rayures puisqu'il dissimule en partie l'animal par des feuilles. Or, les descriptions langagières «ne refusent jamais explicitement de s'engager de cette manière-là $»^{1}$. On peut très bien dire «je n'ai aucun commentaire à faire sur ceci» et poursuivre l'air de rien en donnant son avis malgré tout. Autre exemple typique: mon fils, qui prépare manifestement un cadeau pour la fête des mères, commence par me dire " je ne peux pas te dire quelque chose... », pour ajouter au plus vite « j'ai fait un truc avec des cœurs!». Par ailleurs, dans le langage, décrire quelque chose comme possédant une propriété n'impose aucune restriction quant aux autres propriétés. Alors qu'il en va tout autrement pour la dépiction. Selon Lopes, la totalité des choix et des refus, à l'égard des propriétés d'un sujet que l'image décide de représenter ou d'écarter, constitue l'aspect qu'elle présente de son sujet.

Je conçois l'aspect iconique (pictorial aspect) comme un modèle de saillance visuelle (visual salience), un modèle, aussi bien de ce que l'image laisse de côté que de ce qu'elle inclut. Clairement, certains modèles d'engagement et de non-engagement sont caractéristiques des images. Quels sont-ils ? Qu'estce, pour une image, que de présenter un aspect de son sujet ? ${ }^{2}$.

Bien souvent, la sélectivité concerne des modes de représentation spatiale, même si la texture ou les couleurs peuvent aussi induire certains types d'engagements parfois exclusifs (les images égyptiennes, byzantines, albertiennes, cubistes, etc. ne possèdent évidemment pas les mêmes caractéristiques formelles). Pourtant, on peut d'abord écarter l'idée qu'il s'agirait seulement, pour l'image, d'offrir et même d'imposer un "point de vue » (même s'il s'agit là d'une option tentante pour comprendre les aspects décrits par Lopes). L'aspect iconique - comme modélisation de l'engagement ou du refus à l'égard de certaines propriétés possibles de l'image — ne se réduit pas au seul choix du point de vue. Preuve en est le fait que certaines images prennent le parti de combiner des points de vue pourtant difficilement combinables dans l'expérience de perception naturelle :

Certaines images représentent des objets depuis des points de vue indéterminés, rendant ainsi leurs engagements ou non-engagements explicites cohérents avec ceux des expériences visuelles des objets vus depuis n'importe quel point de vue. À titre d'exemple on songe au cas connu de la figure accroupie

${ }^{1}$ Ibid., p. 119 ; trad. fr. p. 145.

${ }^{2}$ Ibid., p. 119 ; trad. fr. p. 146. 
dans l'angle inférieur droit des Demoiselles d'Avignon qui est représentée comme si elle était vue de dos aussi bien que de face ${ }^{1}$.

De la même manière, les choix qui déterminent la dépiction ne se réduisent pas à ceux observés dans la vision naturelle : les images ont par exemple la capacité de produire en deux dimensions ce qui serait paradoxal - et donc impossible - dans une expérience ordinaire non-iconique (ex. Fausse perspective de Hogarth ou Cascade de Escher). La variété des dispositifs possibles (la variété des styles) et la diversité des aspects représentés dément selon Lopes la règle d'Alberti selon laquelle « les images devraient présenter les objets comme ayant des propriétés qu'on peut voir qu'ils ont quand on les regarde depuis un certain point de vue $»^{2}$.

\section{La reconnaissance d'aspect (ou reconnaissance iconique)}

Pour résumer ce dernier point, la sélectivité - comme marque structurelle distinctive de la dépiction (qui doit choisir de «s'engager sur» ou de nier explicitement certains aspects du monde) - détermine ce que Lopes nomme l'aspect iconique, celui-ci appelant, de la part du spectateur, la reconnaissance d'aspect (c'est-à-dire un travail de compréhension du système ou du modèle de l'image). Il y a donc des normes de composition qui affectent directement le sens de l'image en tant qu'elles soulignent et imposent différents aspects du monde visible au détriment et à l'exclusion d'autres aspects.

Ce que Lopes appelle le «contenu» de l'image (image content) ne peut ni être exclusivement saisi par la perception, ni seulement compris à partir d'aptitudes cognitives (ces deux voies ont été identiquement repoussées) : le contenu de l'image est aspectuel — il s'agit d'un troisième terme qui se situe dans la zone d'équilibre entre la dimension matérielle et la dimension conceptuelle (ou représentationnelle) de l'image.

Le « contenu » d'une image est à distinguer à la fois de son dessin (design) et de son sujet (content). Le contenu d'une représentation, quelle qu'elle soit, mentale, linguistique, iconique, ou bien musicale, consiste dans les propriétés

${ }^{1}$ Ibid., p. 120-121 ; trad. fr. p. 147.

${ }^{2}$ Ibid., p. 124 ; trad. fr. p. 149. 
qu'elle assigne au monde. Le contenu iconique (pictorial content), par extension, est constitué par les propriétés qu'une image assigne au monde ${ }^{1}$.

Il ne s'agit pas du dessin (au sens de « facture »), il ne s'agit pas du sujet (au sens iconologique), mais de l'articulation de l'un à l'autre, établie en fonction des choix opérés par l'image de représenter tel ou tel aspect (et de nier tel autre). Et - à mes yeux — seule une compréhension approfondie des modalités de cette articulation justifie l'intérêt de cette théorie. Sinon, on enfonce des portes ouvertes: l'idée que la compréhension des images mobilise autant la perception que les compétences cognitives étant tout de même assez commune. Or, traditionnellement, et en tout cas dans l'iconologie, plutôt que de tenter l'articulation serrée de la perception et de la cognition dans un travail commun provoqué par l'expérience artistique, on a pensé leur superposition hiérarchisée, l'une s'appuyant sur l'autre pour s'élever.

Cette théorie implique par ailleurs que l'image soit toujours comprise dans sa relation avec un système. Elle ouvre par conséquent la possibilité d'une taxinomie des systèmes de représentation car, en droit, on pourrait considérer qu'il existe autant de systèmes que d'artistes. Lopes identifie quelques-uns de ces systèmes, en les concevant suffisamment larges et recouvrant parfois des époques entières, sans prétendre à l'exhaustivité, mais en apportant la démonstration de la variété de leurs logiques. On peut en citer ici quelques-uns : la dépiction albertienne (tout dans la représentation doit être visible depuis un unique point de vue), la dépiction en perspective curvilinéaire (toutes les faces des objets représentés sont visibles sauf leur dos), la dépiction axonométrique (tout est présenté en parallèle sans profondeur), le style dédoublé (les deux côtés — d'un animal par exemple sont présentés symétriquement), etc.

\section{Les images nous instruisent (conclusion)}

Je reviens en conclusion à la question abordée en ouverture : l'iconologie estelle une théorie élitiste de l'approche des œuvres d'art et plus largement des artefacts visuels? Il me semble a priori que la théorie que je viens de présenter l'est beaucoup moins, et elle m'intéresse pour cette raison précise. L'apport majeur et le plus original de la proposition de Lopes pourrait être le suivant: l'idée de la reconnaissance d'aspect comme identification par le

${ }^{1}$ Ibid., p. 3-4 ; trad. fr. p. 24. 
spectateur d'un système de représentation précis suppose une fréquentation des images suffisamment intense et assidue que pour pouvoir, par le service de la mémoire, informer et augmenter notre capacité de compréhension des images. Et s'il semble évident que la fréquentation régulière d'images (en tout cas artistiques) reste sociologiquement déterminée, on peut néanmoins espérer qu'une familiarité puisse s'installer :

La capacité à reconnaître visuellement des objets implique et met à disposition un riche fond d'information visuelle acquise au cours de rencontres antérieures avec ces mêmes objets ${ }^{1}$.

Autrement dit, sur base de rencontres perceptives avec les images, nous développons des capacités de reconnaissance mobilisables en d'autres occasions. La théorie de Lopes est utile pour expliquer l'acquisition de compétences (capacités de reconnaître) par les images, et pour montrer comment les images elles-mêmes donnent les clés qui permettent d'installer progressivement ces compétences.

Être capable de reconnaître quelque chose qui est présenté sous un aspect iconique typique d'un système n'est pas suffisant pour être capable de reconnaître n'importe quoi qui se présente sous un autre aspect typique d'un autre système. Être compétent en systèmes albertiens ne garantit pas qu'on soit compétent dans le système du style dédoublé, ou vice versa. Mais une fois qu'un spectateur a acquis la capacité de reconnaître certains objets dont il est familier, quand ils sont présentés sous des aspects du style dédoublé, il est alors, en principe, capable de reconnaître n'importe quel objet dont il est familier sous des types d'aspects du style dédoublé. La compétence en matière de dépiction est acquise pour un système à la fois, plutôt que pour une image à la fois ${ }^{2}$.

On peut avoir une idée assez intuitive de ce phénomène de constitution progressive d'aptitudes visuelles en considérant par exemple la bande dessinée destinée à un public très jeune et non averti (par ex. Petit Poilu ${ }^{3}$ ). L'expérience de lecture répétée (et dans ce cas-ci accompagnée par l'adulte qui raconte) permet un apprentissage progressif — par l'image — des codes de la bande dessinée : distribution de l'action en différentes séquences séparées, articulation des vignettes, temporalité reconstituée, etc. La reconnaissance

${ }^{1}$ Ibid., p. 44 ; trad. fr. p. 66.

${ }^{2}$ Ibid., p. 148-149; trad. fr. p. 178.

${ }^{3}$ Bande dessinée à grand succès conçue par deux artistes belges, Pierre Bailly (dessin) et Céline Fraipont (scénario). Cf. http://www.petitpoilu.com 
d'aspect est déclenchée par le dispositif à la fois typique d'un style et original (dispositif volontairement muet dans le cas de Petit Poilu) : l'enfant apprend à reconnaître les choses qu'il connaît déjà (le rituel de départ à l'école, par exemple) telles que traitées dans un système formel singulier. Il apprend par là à maîtriser la dépiction, au moins virtuellement, puisqu'il s'ajuste à elle.

En particulier, les spectateurs interprètent les images en reconnaissant leurs sujets sous les aspects qu'elles présentent. (...) Les images sont des prothèses visuelles; elles étendent le système informationnel en rassemblant, stockant, et transmettant de l'information visuelle à propos de leurs sujets, de manières qui dépendent de notre capacité à identifier les choses d'après leur appartenance, et qui accroissent aussi cette capacité ${ }^{1}$.

Il ne s'agit donc pas, comme une lecture plate de Panofsky aurait pu le laisser penser à propos de l'iconologie, de convoquer des connaissances antérieures, précédemment acquises, ou de mobiliser - pour dégager la signification la plus haute de l'œuvre - des connaissances extérieures à l'expérience sensible de rencontre avec l'œuvre. Il s'agit plutôt de se laisser instruire par les œuvres elles-mêmes, puisque "l'interprétation des images dépend de l'exercice de capacités de reconnaissance relatives aux aspects (interpreting pictures depends on the exercise of aspect-relative recognition abilities) ${ }^{2}$. Pour le dire encore autrement: les images ne nous renvoient pas à des savoirs extra-visuels ; elles incarnent (portent en leur matérialité) de l'information, non pas seulement à propos du monde, mais à propos de la manière dont leurs contenus permettent de reconnaître leurs sujets.

\section{Bibliographie}

Belting H., «Echte Bilder und falsche Körper - Irrtümer über die Zukunft des Menschen », in Christa Maar/Hubert Burda (ed.), Iconic turn. Die neue Macht der Bilder, DuMont, 2004.

Boehm G., «Die Bilderfrage », Was ist ein Bild?, ed. Gottfried Boehm, Munich 1994.

—, « Jenseits der Sprache? Anmerkungen zur Logik der Bilder », Iconic Turn. Die Neue Macht der Bilder, ed. Christa Maar and Hubert Burda, Cologne, 2004.

Bourdieu P., La distinction. Critique sociale du jugement, Paris, Minuit, coll. « Le sens commun », 1979.

${ }^{1}$ D. Lopes, Understanding Pictures, op. cit., p. 144 ; trad. fr. p. 173-174.

${ }^{2}$ D. Lopes, Understanding Pictures, op. cit., p. 149 ; trad. fr. p. 179. 
Bredekamp H., « Drehmomente - Merkmale und Ansprüche des iconic turn », in C. Maar, H. Burda (ed.), Iconic turn. Die neue Macht der Bilder, DuMont, 2004.

Didi-Huberman G., Devant l'image, Paris, Minuit, 1990.

Dretske F.I., Knowledge and the Flow of Information, Cambridge, The MIT Press/Bradford Books, 1981.

Goodman N., Languages of Art. An Approach to a Theory of Symbols, Indianapolis, Bobbs-Merrill, 1968 (2d ed. Indianapolis, Hackett, 1976).

Kulvicki J.V., On Images. Their Structure and Content, Oxford University Press, 2009.

Lopes D., « Picture this: Image-based Demonstratives », in Catharine Abell, Katerina Bantinaki (ed.), Philosophical Perspectives on Depiction, Oxford University Press, 2010.

—, Sight and Sensibility, Oxford University Press, 2005.

-, Understanding Pictures, Oxford Scholarship, 1996. Traduction française de L. Blanc-Benon: Comprendre les images: une théorie de la représentation iconique, Rennes, PUR, coll. « Aesthetica », 2014.

Merleau-Ponty M., La prose du monde, texte établi et présenté par C. Lefort, Paris, Gallimard, 1969.

Mitchell W.J.T., Iconology : Image, Text, Ideology, Chicago, University of Chicago Press, 1986.

-, Picture Theory. Essays on Verbal and Visual Representation, Chicago, University of Chicago Press, 1994.

- What do Pictures Want? The Lives and Loves of Images, Chicago, University of Chicago Press, 2005.

Panofsky E., Architecture gothique et pensée scolastique (1951), Paris, Minuit, coll. «Le sens commun », 1967.

—, Essais d'iconologie: thèmes humanistes dans l'art de la Renaissance (Studies in Iconology, 1939), trad. fr. C. Herbette et B. Teyssèdre, Paris, Gallimard, 1967.

-, L'Euvre d'art et ses significations (Meaning in the Visual Arts, 1955), trad. fr. par M. et B. Teyssèdre, Paris, Gallimard, 1969.

Pasolini P.P., Lettres luthériennes. Petit traité pédagogique (1976), trad. fr. A.R. Pullberg, Paris, Seuil, 2000.

Peacocke C., « Perceptual content », in J. Almog, J. Perry, H. K. Wettstein, Themes from Kaplan, New York, Oxford University Press, 1983.

Rieber A., Art, histoire et signification. Un essai d'épistémologie d'histoire de l'art autour de l'iconologie d'Erwin Panofsky, Paris, L'Harmattan, 2012.

Sachs-Hombach K., « Das Bild in der Spannung von perzeptuellen und semiotischen Determinanten », in K. Sachs-Hombach, Bildwissenschaft. Zwischen Reflexion und Anwendung, Halem, 2005.

Sachs-Hombach K., Schirra J.R.J., « Medientheorie, visuelle Kultur und Bildanthropologie », in K. Sachs-Hombach, Bildtheorien. Anthropologische und kulturelle Grundlagen des visualistic turn, Suhrkamp, 2009.

Van Fraasen B.C., Scientific Representation, Oxford University Press, 2008. 
Waldenfels B., « Ordnungen des Sichtbaren », in G. Boehm, Was ist ein Bild?, Fink, 1994.

Wiesing L., «Methoden der Bildwissenschaft», in K. Sachs-Hombach, Bildwissenschaft. Zwischen Reflexion und Anwendung, Halem, 2005.

—, «Sind Bilder Zeichen? », in K. Sachs-Hombach, K. Rehkämper, Bild Bildwahrnehmung - Bildverarbeitung, DUV, 1998.

Wolheim R., « Voir-comme, Voir-dans et la représentation picturale », in L'art et ses objets, trad. R. Crevier, Paris, Aubier, 1994.

—, Painting as an Art, Princeton, Princeton University Press, 1987. 